\title{
Flow-Induced Helical Coiling of Semiflexible Polymers in Structured Microchannels
}

\author{
Raghunath Chelakkot, ${ }^{1,2}$ Roland G. Winkler, ${ }^{1}$ and Gerhard Gompper ${ }^{1}$ \\ ${ }^{1}$ Theoretical Soft Matter and Biophysics, Institute of Complex Systems and Institute for Advanced Simulation, \\ Forschungszentrum Jülich, 52425 Jülich, Germany \\ ${ }^{2}$ Martin Fisher School of Physics, Brandeis University, Waltham, Massachusetts 02453, USA
}

(Received 3 August 2012; published 23 October 2012)

\begin{abstract}
The conformations of semiflexible (bio)polymers are studied in flow-through geometrically structured microchannels. Using mesoscale hydrodynamics simulations, we show that the polymer undergoes a rodto-helix transition as it moves from the narrow high-velocity region into the wide low-velocity region of the channel. The transient helix formation is the result of a nonequilibrium and nonstationary buckling transition of the semiflexible polymer, which is subjected to a compressive force originating from the fluid-velocity variation in the channel. The helix properties depend on the diameter ratio of the channel, the polymer bending rigidity, and the flow strength.
\end{abstract}

DOI: 10.1103/PhysRevLett.109.178101

PACS numbers: 87.16.Ka, 83.50.-v

Buckling is a common phenomena of slender bodies, such as long filaments and thin sheets, under an external load. Examples range from microscopic to macroscopic length scales and include actin filaments, vaulting poles, virus capsids, and tectonic plates. Specifically, slender rods or semiflexible (bio)polymers exhibit a buckling instability under compression, when the load exceeds a critical value [1-3]. In the simplest situation of Euler buckling of a rod, the symmetry $\left(\mathrm{O}_{2}\right.$ symmetry along the rod axis) is broken by buckling perpendicular to its axis in an arbitrary direction $[4,5]$. Since buckling is of such fundamental importance, it has received persistent attention over centuries.

Typically, buckling transitions are considered under equilibrium conditions. For macromolecular and biological filaments with typical length scales of nanometers to micrometers, which we are interested in, thermal fluctuations broaden the buckling transition and generate a smooth crossover from the unbuckled to the buckled state [1-3]. However, very little attention has been payed to conformational instabilities far from equilibrium $[5,6]$, which exhibit qualitatively new features compared to equilibrium ones. Here, the transport of semiflexible polymers in microchannels and capillaries presents a new opportunity to study the nonequilibrium behavior of such filaments [7-9]. Vice versa, a detailed understanding of the dynamical process involved in such a transport is of paramount importance in many applications. This applies, in particular, to many biologically relevant polymers, such as DNA, actin filaments, and microtubules, which are semiflexible; an example is DNA sorting in microchannels [10-12].

Nonequilibrium instabilities can appear under various conditions. Here, we investigate flow fields of spatially varying flow strength. Such a situation is easily realized in flows through spatially structured microchannels, e.g., in a region where the channel width changes from narrow to wide (see Fig. 1). By mesoscale hydrodynamic simulations of a semiflexible polymer in such a microchannel, we observe a buckling of the polymer as it enters the wider channel section. Buckling is often the first step in the formation of more complex structures $[13,14]$. Indeed, we observe that buckling is followed by a flow-induced helical coiling of the polymer.

Helices are a preferred shape in nature. They are frequently adopted in biological systems such as proteins, they have been shown to be close to optimal packing under the restriction of their own volume and finite length [13], they can arise for entropic reasons in the presence of depletant molecules [14], and bacteria swim by rotating helical filaments [15]. Helices are also formed by falling ropes and falling fluid filaments [16,17], seen transiently in homopolymer collapse from an extended conformation [18], or induced by electrostatic interactions in polyelectrolytes [19]. However, as we will explain, the helix formation process in microchannel flow is different from previously studied cases because it occurs at small Reynolds numbers, in the absence of attractive interactions, and is mainly due to solvent friction. (a)

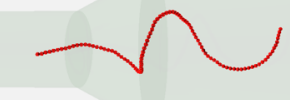

(b)

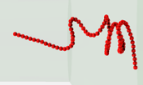

FIG. 1 (color online). Polymer conformations in channels with the diameter ratios (a) $D_{w} / D_{n}=2$ and (b) $D_{w} / D_{n}=4$ for the flow strength $\mathrm{Pe} \simeq 550$ and $L / L_{p}=1$. See also Movies $\mathrm{S} 1$ and $\mathrm{S} 2$ in the Supplemental Material [27]. 
We apply a hybrid simulation method, in which molecular dynamics simulations for semiflexible polymers are combined with a mesoscale simulation method, the multiparticle collision dynamics (MPC) approach, for the solvent $[20,21]$. In the MPC algorithm, the fluid is described by a set of $N$ point particles of mass $m$, with velocities determined by a stochastic process. The particle dynamics evolves in two steps. In the streaming step, the solvent particles move ballistically for a time interval $h$. In the collision step, particles are sorted into the cells of a cubic lattice of lattice constant $a$ and their relative velocities, with respect to the center-of-mass velocity of each cell, are rotated around a randomly oriented axis by an angle $\alpha$. For every cell, mass and momentum are conserved, which leads to the buildup of hydrodynamic interactions (HI) between the fluid particles; at the same time thermal fluctuations are taken into account [20-22].

The fluid is confined in a cylindrical channel of diameter $D_{w}$, with periodic constrictions of smaller diameter $D_{n}$ (see Fig. 1). In order to create a smooth laminar flow, there is a segment between the narrow and wide parts, in which the diameter interpolates linearly between $D_{n}$ and $D_{w}$. The length of the constriction is larger than the polymer contour length $L$. No-slip boundary conditions are imposed on the channel walls by the bounce-back rule and virtual-wall particles [21]. Flow is induced by a gravitational force $(\mathrm{mg})$ acting on every fluid particle in the direction of the channel axis, which yields parabolic flow profiles; in the parts connecting the narrow and wide segments, there is also a radial flow component. The difference in velocity between the narrow and wide parts of the channel is $\left|v_{n}\right|-\left|v_{w}\right|=$ $\left|v_{n}\right|\left(1-D_{n}^{2} / D_{w}^{2}\right)=\Delta v$, where $v_{w}$ and $v_{n}$ denote the velocities in the wide and narrow segments, respectively.

The polymer is represented by a bead-spring model, in which $N_{m}$ monomers, each of mass $M$, are linearly connected by harmonic springs of equilibrium length $b$ [23]. Excluded volume interactions are taken into account by a purely repulsive (truncated and shifted) Lennard-Jones potential [21,23]. Additionally, a three-body potential is applied to capture bending stiffness, with the bending rigidity $\kappa$ [7].

The polymer-fluid interaction is taken into account by including the monomers in the collision step [21]. We employ the MPC parameters $h=0.1 \sqrt{m a^{2} / k_{B} T}, \alpha=130^{\circ}$, the mean number of particles per collision cell $\langle N\rangle=10$, and the fluid mass density $\varrho=\langle N\rangle m / a^{3}$. For the polymer, we set $M=m\langle N\rangle, b=a$, and the Lennard-Jones parameters $\sigma=b$ and $\epsilon / k_{B} T=1$. If not otherwise indicated, the polymer length is $L / b=N_{m}-1=99$. To maintain a constant temperature, a local velocity scaling algorithm is applied, which yields Maxwell-Boltzmann distributed velocities [24]. We characterize the strength of the flow by the Peclet number Pe $=\dot{\gamma} \tau$, where $\dot{\gamma}=g \varrho D_{n} /(4 \eta)$ is the shear rate at the cylinder wall and $\tau$ is the longest relaxation time of a semiflexible polymer [25]. On the basis of a semiflexible polymer model, we find the relaxation time $\tau / \sqrt{m a^{2} / k_{B} T} \approx 1.4 \times 10^{6} L / L_{p}$ for the above parameters, where $L_{p}=\kappa /\left(k_{B} T\right)$ is the persistence length of the polymer.

Figure 1 shows snapshots of polymer conformations when the polymer leaves the constriction and enters the wider part of the channel. Inside the constriction, the polymer conformations are rodlike with small fluctuations. When the polymer is ejected from the constriction, it enters a region where the fluid velocity decreases. Hence, it is subjected to a compressive force as a result of the difference in fluid velocity, and the polymer undergoes conformational changes. With increasing flow strength, we find a gradual change in transient polymer conformations from (bend) rods to helical shapes. This helical conformation is a novel feature for a semiflexible polymer and occurs by spontaneous symmetry breaking. Simulations for various channel diameters $D_{w}$, but fixed constriction diameter $D_{n}$, show that the helix diameter strongly depends on $D_{w}$ and decreases with increasing channel diameter ratio $D_{w} / D_{n}$; see Fig. 1. We attribute this behavior to the larger compressive force due to the larger difference $\Delta v$ in fluid velocities for larger $D_{w} / D_{n}$ ratios. It is evident from the snapshots, in particular in Fig. 1(b), that the polymers assume helical shapes without any direct interaction with the channel walls. Hence, any qualitative influence of confinement can be unequivocally ruled out as the dominating mechanism.

In order to understand the underlying mechanisms and driving forces, we first study the influence of hydrodynamic interactions. Simulations with turned-off hydrodynamic correlations using the Brownian MPC approach [21], where each monomer independently performs stochastic collisions with uncorrelated fluid particles subject to a parabolic flow profile, provide the same qualitative behavior. Hence, the rod-to-helix transition is not caused by hydrodynamic interactions, although substantial quantitative differences in helical properties are observed.

To quantify the helical conformations, we introduce the torsional order parameter $U_{T}=\sum_{i=1}^{N_{m}-1} \cos \gamma_{i}$, where the angle $\gamma_{i}$ is defined by the three subsequent bond vectors $\boldsymbol{R}_{i-1}, \boldsymbol{R}_{i}$, and $\boldsymbol{R}_{i+1}$, with

$$
\cos \gamma_{i}=\frac{\left(\boldsymbol{R}_{i-1} \times \boldsymbol{R}_{i}\right) \cdot\left(\boldsymbol{R}_{i} \times \boldsymbol{R}_{i+1}\right)}{\left|\boldsymbol{R}_{i-1} \times \boldsymbol{R}_{i}\right|\left|\boldsymbol{R}_{i} \times \boldsymbol{R}_{i+1}\right|} .
$$

$U_{T}$ is displayed in Fig. 2(a) for the persistence length $L_{p}=4 L . U_{T}$ increases as the polymer forms a helix when it is ejected from the constriction and decreases again as the polymer relaxes back to its original straight configuration. Simulations without hydrodynamic interactions display a very similar behavior except that the values of $U_{T}$ are significantly larger; this is due to the lack of fluid being dragged along by the polymer segments.

Figure 2(b) presents the maximum values $U_{T}^{m}$ of the average order parameter $\left\langle U_{T}(z)\right\rangle$ for various stiffnesses in 

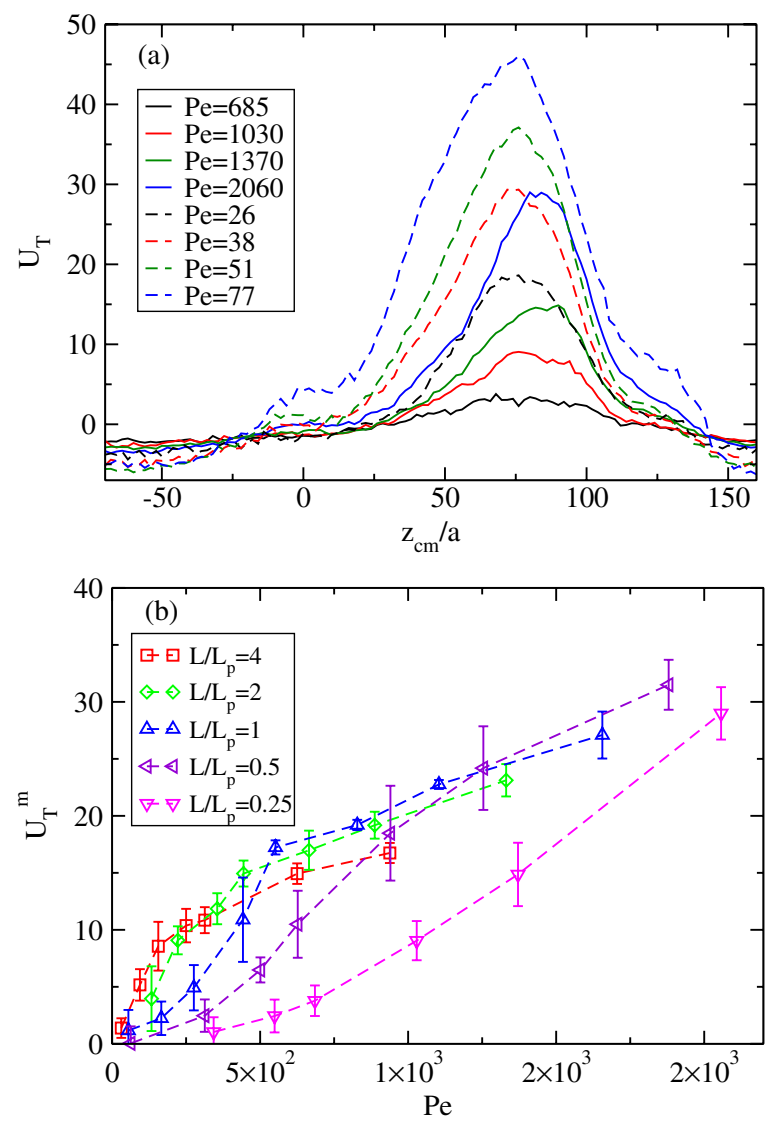

FIG. 2 (color online). (a) Order parameter $U_{T}$ as function of the axial position of the polymer center of mass for systems with (solid lines) and without (dashed lines) hydrodynamic interactions. The flow strengths are $\mathrm{Pe}=685,1030,1370,2060$, (HI) and $\mathrm{Pe}=26,38,51,77$ (no $\mathrm{HI}$ ), with the ratios $L / L_{p}=1 / 4$ and $D_{w} / D_{n}=2$. (b) Peak values $U_{T}^{m}$ of the order parameter $U_{T}$ for the persistence lengths $L / L_{P}=4.0(\square), 2.0(\diamond), 1.0(\triangle), 0.5$ $(\triangleleft)$, and $0.25(\nabla)$. The results are for the MPC fluid with $D_{w} / D_{n}=2$.

the range $1 / 4 \leq L_{p} / L \leq 4$. The parameter of more flexible polymers increases faster than that of stiffer ones. All peak values seem to saturate at high Peclet numbers, where the saturation value increases with increasing stiffness. Within a certain range of Peclet numbers, a universal dependence of $U_{T}^{m}$ appears independent of stiffness.

To elucidate the factors that cause helical coiling of a polymer under nonhomogeneous compressive forces, we consider a simpler system, where a polymer in a viscous fluid is driven by a rigid wall (penetrable to the fluid), which moves with the constant velocity $v_{w}$. Thermal fluctuations and hydrodynamics interactions are omitted for simplicity. The planar wall is parallel to the $x y$ plane and perpendicular to the polymer, which is oriented initially along the $z$ direction with one end in contact with the wall. The polymer dynamics is governed by

$$
M \ddot{\boldsymbol{r}}_{i}=-\Gamma \dot{\boldsymbol{r}}_{i}+\boldsymbol{F}_{i}^{w} \Theta\left(z_{w}-z_{i}\right)+\boldsymbol{F}_{i}^{l}+\boldsymbol{F}_{i}^{b},
$$
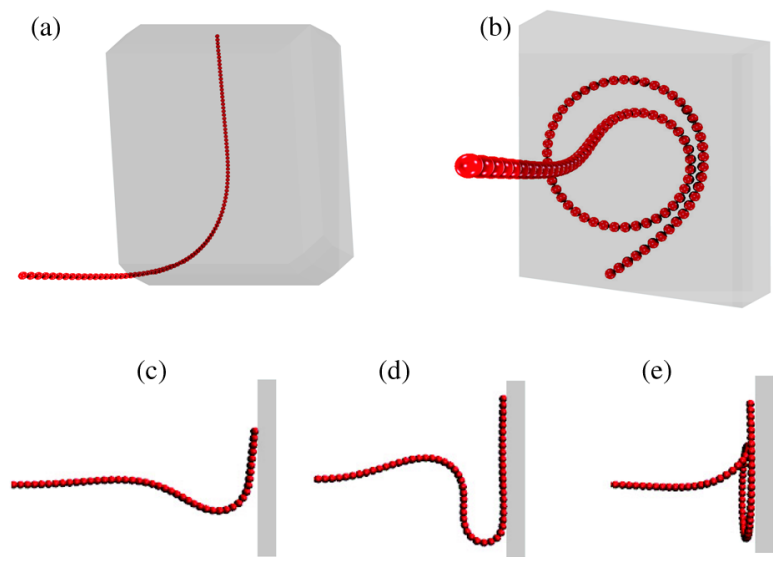

FIG. 3 (color online). Typical configurations of buckled semiflexible polymers with $L_{p} / L=1.0$. The rigid substrate is indicated by the gray area. The polymer length is $N_{m}=100$ and the force is $F /\left(k_{B} T / a\right)=100$. The viscous drag coefficient is (a) $\Gamma / \sqrt{m k_{B} T / b^{2}}=1.0$ and (b) 50.0. (c)-(e) Sequence of images illustrating the helical coiling of the semiflexible polymer during the initial phase. See also Movies S3 and S4 in the Supplemental Material [27].

where $\boldsymbol{r}_{i}$ is the position of monomer $i, z_{w}$ is the position of the wall, $\Theta(z)$ is the Heaviside step function, $\boldsymbol{F}_{i}^{l}$ and $\boldsymbol{F}_{i}^{b}$ are the bond and bending forces, respectively, and $\Gamma$ is the monomer friction coefficient. $\boldsymbol{F}_{i}^{w}=F \boldsymbol{e}_{z}$, where $\boldsymbol{e}_{z}$ is the unit vector along the $z$ axis, describes the force of the wall on monomer $i$ when it is in contact with the wall. Equation (2) is solved numerically for a polymer with $L_{p} / L=1$.

For small $\Gamma$, less than a threshold $\Gamma_{c}^{(1)}$, frictional dissipation is weak and the polymer moves with the wall without any significant conformational changes. For fixed $\boldsymbol{v}_{w}$, the behavior changes qualitatively when $\Gamma$ exceeds $\Gamma_{c}^{(1)}$, because the compressive force due to polymer friction now leads to a buckling transition, similar to classical Euler buckling. As the wall moves further, the part of polymer in contact with the wall aligns with the wall and moves along the wall, whereas the part further away from the wall moves in the same direction, but at a smaller velocity [cf. Fig. 3(a)]. Ultimately, the entire polymer is stretched out parallel to the wall. With further increasing $\Gamma$, beyond a threshold $\Gamma_{c}^{(2)}$, we observe a distinctly different behavior, as the polymer now exhibits a transition to a coiled state [cf. Fig. 3(b)]. As displayed in Fig. 4, the bending energy $U_{b}$ of the polymer exhibits two different regimes as function of time for such systems. Initially, $U_{b}$ increases by local Euler-like buckling of the polymer over a length scale much smaller than its length [cf. Figs. 3(c) and 4(a)]. When the bending energy exceeds a certain value, the polymer undergoes a conformational change to a coiled state near the wall [Figs. 3(d) and 3(e)], and $U_{b}$ drops significantly. For later times, the polymer continues coiling until the entire chain is coiled up at the wall. 

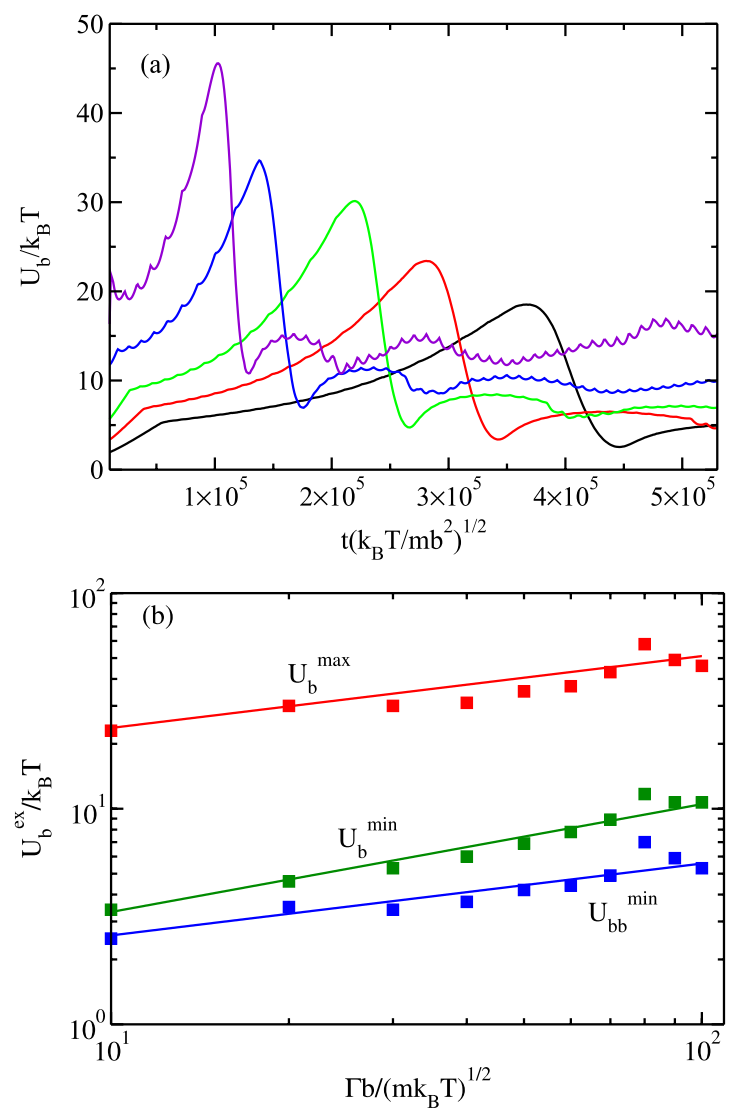

FIG. 4 (color online). (a) Time-dependent bending energy $U_{b}$ of a semiflexible polymer with $L / L_{p}=1.0$ pushed forward by a rigid wall moving with velocity $v_{w}=0.1 \sqrt{k_{B} T / m}$. Curves from left to right correspond to decreasing drag coefficients $\Gamma / \sqrt{m k_{B} T / b^{2}}=100,50,20,10,5$. (b) Dependence of the maximum bending energy $U_{b}^{\max }$ (top), the minimum energy after the drop $U_{b}^{\min }$ (middle), and the minimum bulk energy $U_{b b}^{\min }$ (bottom) on friction. The slopes of the straight lines are 1/3 (top, bottom) and 1/2 (middle).

The dependence of the peak value of the bending energy on the wall velocity and the friction coefficient $\Gamma$ can be understood from some scaling arguments. For $\Gamma<\Gamma_{c}^{(1)}$, the motion of the polymer through the viscous medium generates a inhomogeneous (negative) tension $\sigma(s)=\Gamma v_{w}(L-s)$, where $s$ is the arc length measured from the polymer end at the wall. For a homogeneous tension $\sigma_{0}$, buckling theory predicts an instability with a fastest growing mode with wave vector $q \sim \sqrt{\sigma_{0} / \kappa}$; hence, buckling occurs when $q>2 \pi / L$. If we approximate the tension by its average $\sigma_{0}=\Gamma v_{w} L / 2$, we obtain $\Gamma_{c}^{(1)} \sim \kappa v_{w}^{-1} L^{-3}$.

For friction coefficients $\Gamma>\Gamma_{c}^{(2)}$, initial buckling occurs at the near-wall end of the polymer [Fig. 3(c)]. The bending energy of the buckled part of contour length $L_{c}$ has the local curvature $1 / R(s)$, and hence, the bending energy is $U_{b} \sim \kappa \int_{0}^{L_{c}} R^{-2} d s$. Since there is only local bending of the polymer over a length scale $L_{c} \approx R$, we find $U_{b} \sim \kappa / R$ by replacing $R(s)$ with a characteristic value $R$. The resulting bending force $F_{b}=-\kappa / R^{2}$ is balanced by the frictional force over the same length scale, i.e., $F_{f}=\Gamma v_{w} R$, which implies a curvature radius

$$
R \sim\left[\kappa /\left(\Gamma v_{w}\right)\right]^{1 / 3},
$$

and a bending energy

$$
U_{b} \sim \kappa^{2 / 3}\left(\Gamma v_{w}\right)^{1 / 3}
$$

During the transition from local buckling to circular coiling, the curvature of the buckled part decreases, which explains the drop in bending energy [Figs. 3(d) and 3(e)]. At later times, during the stationary coiling process, the dynamics is determined by two contributions: the bending energy of a part $L_{c}$ not in contact with the wall $U_{b b}$ (bulk part) and a part of circular shape at the wall. For the bulk part of the bending energy, the same argument as in the derivation of Eqs. (3) and (4) applies. Hence, we expect to find the same scaling relation for bending in the bulk.

Figure 4(b) hows the scaling behavior with respect to friction of the maximum bending energy $U_{b}^{\max }$ of Fig. 4(a). This energy nicely follows the scaling prediction (4). To characterize the quasistationary behavior of the bending energy for long times, we determine the minimum energy $U_{b b}^{\min }$ of the bulk part of the bending energy after the steep drop of $U_{b}$. The numerical simulations show that the bulk bending energy dominates over the wall part for small friction coefficients. At the larger $\Gamma$ the two contributions become comparable. As shown in Fig. 4(b), $U_{b b}^{\min }$ also follows the scaling relation (4), whereas the total minimum energy exhibits the stronger dependence $\Gamma^{1 / 2}$.

The buckling and helical coiling of a polymer under flow in a structured microchannel and a polymer pushed forward by a wall in a viscous medium is governed by very similar mechanisms. In contrast to the moving-wall model, the polymer in the structured microchannel does not exhibit circular collapsed conformations. Indeed, the compressive force due to the fluid velocity difference is less restrictive, because the coiled part of the polymer can relax more easily. The observed behavior differs from classical Euler buckling in several respects. First, the buckling force appears due to a nonequilibrium and nonstationary dynamical process. Second, buckling is initiated locally. This is a consequence of the frictional force, which increases with the length of the considered polymer part. Third, a transition to helical coiling occurs when the local tilt angle exceeds a certain value.

Thus, helical coiling is expected to be a generic phenomenon of polymer motion in nonhomogeneous viscous environments. Actin filaments [8] in structured microchannels [26] seem to be an optimal system to observe the helical-coiling transition experimentally. 
[1] K. Kroy and E. Frey, Phys. Rev. Lett. 77, 306 (1996).

[2] M. Emanuel, H. Mohrbach, M. Sayar, H. Schiessel, and I. M. Kulić, Phys. Rev. E 76, 061907 (2007).

[3] J. Kierfeld, K. Baczynski, P. Gutjahr, T. Kühne, and R. Lipowsky, Soft Matter 6, 5764 (2010).

[4] L.D. Landau and E. M. Lifshitz, Theory of Elasticity (Pergamon, New York, 1986).

[5] L. Golubovic, D. Moldovan, and A. Peredera, Phys. Rev. E 61, 1703 (2000).

[6] Y. Geng, G. Wang, Y. Cong, L. Bai, L. Li, and C. Yang, Macromolecules 42, 4751 (2009).

[7] R. Chelakkot, R. G. Winkler, and G. Gompper, Europhys. Lett. 91, 14001 (2010).

[8] D. Steinhauser, S. Köster, and T. Pfohl, ACS Macro Lett. 1, 541 (2012).

[9] V. Kantsler and R. E. Goldstein, Phys. Rev. Lett. 108, 038103 (2012).

[10] H.-P. Chou, C. Spence, A. Scherer, and S. Quake, Proc. Natl. Acad. Sci. U.S.A. 96, 11 (1999).

[11] K. Jo, D. M. Dhingra, T. Odijk, J.J. de Pablo, M. D. Graham, R. Runnheim, D. Forrest, and D. C. Schwartz, Proc. Natl. Acad. Sci. U.S.A. 104, 2673 (2007).

[12] S. L. Levy and H. G. Craighead, Chem. Soc. Rev. 39, 1133 (2010).

[13] A. Maritan, C. Micheletti, A. Trovato, and J. R. Banavar, Nature (London) 406, 287 (2000).

[14] Y. Snir and R. D. Kamien, Science 307, 1067 (2005).
[15] H. C. Berg and R. A. Anderson, Nature (London) 245, 380 (1973).

[16] L. Mahadevan and J. B. Keller, Proc. R. Soc. A 452, 1679 (1996).

[17] L. Mahadevan, W.S. Ryu, and A.D. T. Samuel, Nature (London) 392, 140 (1998).

[18] S. A. Sabeur, F. Hamdache, and F. Schmid, Phys. Rev. E 77, 020802(R) (2008).

[19] Y. Xu, S. Bolisetty, M. Drechsler, B. Fang, J. Yuan, L. Harnau, M. Ballauff, and A.H. E. Müller, Soft Matter 5, 379 (2009).

[20] R. Kapral, Adv. Chem. Phys. 140, 89 (2008).

[21] G. Gompper, T. Ihle, D. M. Kroll, and R. G. Winkler, Adv. Polym. Sci. 221, 1 (2009).

[22] A. Malevanets and R. Kapral, J. Chem. Phys. 110, 8605 (1999).

[23] C.-C. Huang, R.G. Winkler, G. Sutmann, and G. Gompper, Macromolecules 43, 10107 (2010).

[24] C.-C. Huang, A. Chatterji, G. Sutmann, G. Gompper, R. G. Winkler, J. Comput. Phys. 229, 168 (2010).

[25] L. Harnau, R. G. Winkler, and P. Reineker, J. Chem. Phys. 104, 6355 (1996).

[26] H. Noguchi, G. Gompper, L. Schmid, A. Wixforth, and T. Franke, Europhys. Lett. 89, 28002 (2010).

[27] See Supplemental Material at http://link.aps.org/ supplemental/10.1103/PhysRevLett.109.178101 for simulation animations. 